\title{
Equalização Adaptativa no Domínio da Frequiência para Sistemas com Transmissão em Blocos
}

\author{
Bernardo R. da Costa, Tiago T. V. Vinhoza, Raimundo Sampaio-Neto, Rodrigo C. de Lamare
}

\begin{abstract}
Resumo-Este trabalho investiga e compara diferentes técnicas de equalização no domínio da freqüência para sistemas de uma única portadora SC (single-carrier) e de multiportadoras OFDM (Orthogonal Frequency Division Multiplexing). A transmissão é feita em blocos, assim como seu processamento na recepção. Além da filtragem linear adaptativa no domínio da freqüêencia, que acontece em ambos os sistemas, é também empregado um filtro adaptativo de retorno, no domínio do tempo, para os sistemas SC, e que opera símbolo à símbolo sobre os blocos recebidos. Os resultados obtidos através de simulação mostram que o SC tem desempenho superior ao OFDM.
\end{abstract}

Palavras-Chave-Equalização adaptativa, OFDM, SingleCarrier, Equalização no domínio da freqüiência, Equalização com decisão realimentada.

Abstract-This work presents a comparison between SingleCarrier Frequency Domain Equalization (SC-FDE) and OFDM (Orthogonal Frequency Division Multiplexing). We consider a block transmission and a block processing at the receiver's end. Aside from the linear adaptive equalization in the frequency domain, a adaptive decision feedback equalizer (which operates in a symbol by symbol basis in the time domain) is applied in the SC systems. The zero-padding scheme is used in both systems for the comparisons. Simulation results show that the SC-FDE outperforms OFDM.

Keywords-Adaptive Equalization, OFDM, SingleCarrier, Frequency-Domain Equalization, Decision Feedback Equalização.

\section{INTRODUÇÃO}

Tecnologias de acesso sem fio e de faixa larga, que oferecem altas taxas de transmissão a usuários residenciais e corporativos, são alternativas atraentes e economicamente viáveis às tecnologias de acesso com fio existentes [1]. Nestes ambientes, os efeitos de propagação de múltiplos percursos são bastante severos e capazes de degradar significativamente o desempenho de um sistema de transmissão. Para combater a interferência entre símbolos derivada dos múltiplos percursos de propagação é necessário o emprego de equalizadores. Uma das classes possíveis são os equalizadores MLSD (Maximum Likelihood Sequence Detection) [2], porém apresentam complexidade elevada se comparado aos esquemas de equalização considerados neste trabalho. Dentre as técnicas de combate à interferência entre símbolos mais promissoras e eficientes do ponto de vista econômico e de desempenho, destacam-se a

B. R. da Costa, T. T. V. Vinhoza e R. Sampaio-Neto. Centro de Estudos em Telecomunicações (CETUC), Pontifícia Universidade do Rio deJaneiro (PUC-Rio), Rio de Janeiro (RJ), Brasil. R. C. de Lamare está com o Communications Research Group, Department of Electronics, University of York, York Y010 5DD, United Kingdom. E-mails: costabernardo@gmail.com,\{vinhoza,raimundo\}@ cetuc.pucrio.br, rcd1500@ohm.york.ac.uk. Este trabalho foi parcialmente financiado pelo CNPq. equalização no domínio da freqüência para sistema de única portadora (Frequency-Domain Equalization - SC-FDE) [3] e para os sistemas multiportadoras [4], como por exemplo o OFDM. Em sistemas de transmissão em blocos, os símbolos são agrupados em blocos e a adição de símbolos redundantes (intervalo de guarda) elimina a interferência entre blocos (IEB) e permite o emprego de técnicas eficientes (mais simples) de processamento de sinais na recepção. A literatura nesta área de pesquisa sugere que os sistemas SC-FDE e OFDM apresentam um desempenho e uma complexidade para implementação semelhantes e identifica algumas vantagens em favor do SC no que se refere a sensibilidade a desvios de freqüência e sensibilidade a não-linearidades no transmissor devido a altas excursões de amplitude que ocorrem no sinal de transmissão OFDM e que resultam em um alto valor de PAPR (Peak-toAverage Power Ratio) [1]. Nos últimos anos, a boa relação desempenho/custo das técnicas de equalização SC-FDE tem aumentado significativamente a atividade de pesquisa nesta área. Equalizadores lineares seguidos de um estágio de decisão realimentada (DFE - Decision Feedback Equalizer) são estruturas relativamente simples e obtém ganhos substanciais sobre equalizadores lineares [2]. Uma desvantagem destes equalizadores, porém, é o fenômeno de propagação de erros [2]. Um estágio adicional DFE para sistemas SC-FDE foi considerado em [1] para sistemas com prefixo cíclico (CP), e em [5] para sistemas com zero-padding (ZP). O trabalho em [5], porém, propõe uma solução não-adaptativa e supõe estimação perfeita de canal. Este trabalho é organizado da seguinte forma. A Seção II apresenta o modelo do sistema considerado e os conceitos básicos da transmissão com portadora única (SC) e/ou multi-portadora (OFDM). Considerase apenas o esquema ZP para a faixa de guarda. A Seção III trata da recepção, onde a equalização adaptativa linear é feita no domínio da freqüência, tanto para o OFDM como para o SC-FDE. Esta filtragem será referenciada como Linear Equalization (LE) no SC. A equação para atualização do filtro é descrita, seguindo o método recursivo dos mínimos quadrados (RLS). A Seção IV introduz uma alternativa para refinar as decisões dos sistemas SC-FDE-LE ao fazer uso de um filtro de realimentação, que opera no domínio do tempo. Este sistema será então denominado de SC-FDE-DFE. Neste caso, o filtro adaptativo de retorno e o linear operam simultaneamente e a minimização e a atualização de ambos é feita conjuntamente. A Seção V traz os resultados obtidos por simulação em computador dos sistemas apresentados. Por fim, a seção VI apresenta as conclusões. 


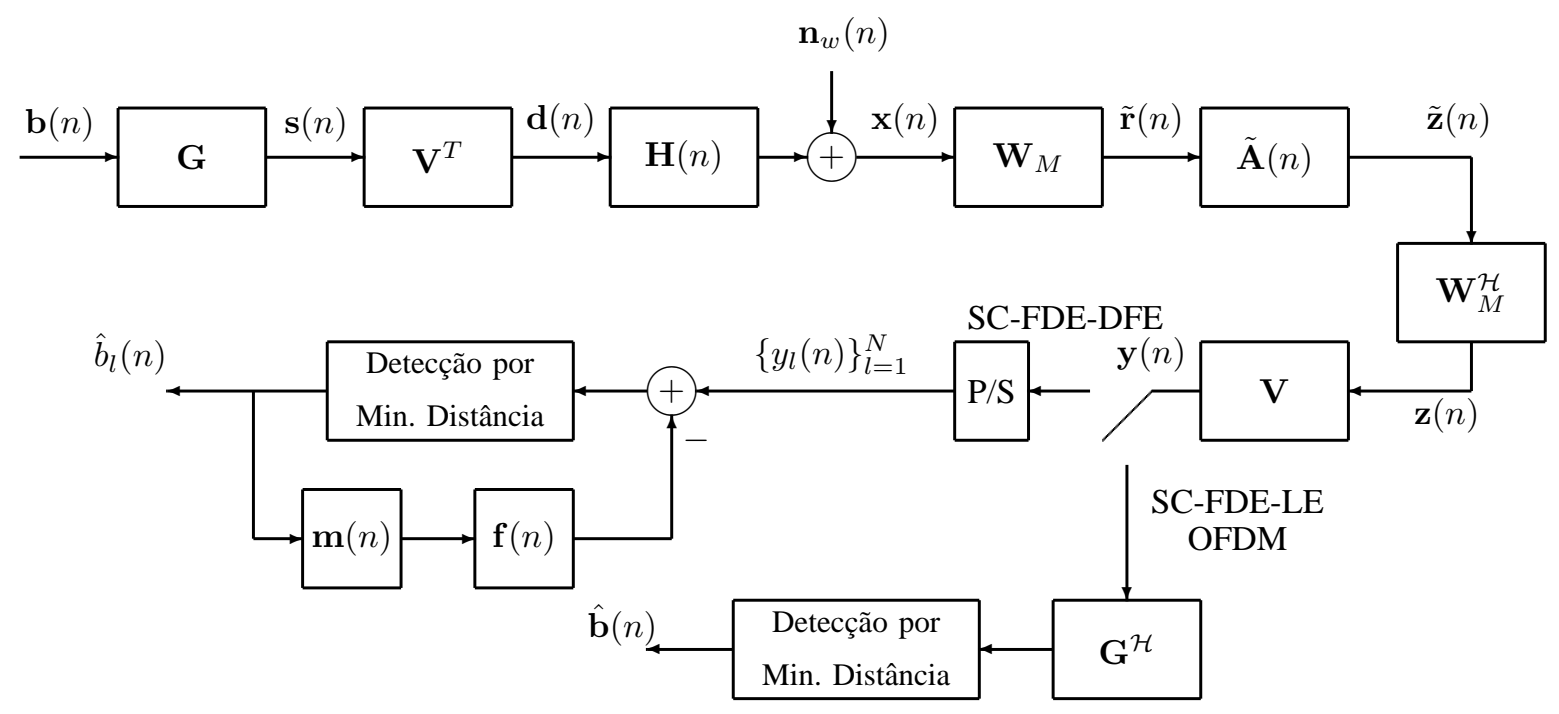

Fig. 1. Diagrama em blocos da estrutura de transmissão/recepção OFDM e SC-FDE

\section{Modelo do Sistema}

Considere o modelo discreto de um sistema de transmissão de blocos mostrado na Fig. 1. Deseja-se transmitir um bloco de informação denotado pelo vetor $\mathbf{b}(n)$ de dimensão $N$.

\section{A. Transmissão}

Neste trabalho, os símbolos contidos em $\mathbf{b}(n)$ representam pontos de uma constelação complexa quaternária (QPSK), de símbolos i.i.d, de média zero e energia unitária. $\mathrm{O}$ bloco $\mathbf{b}(n)$ é então pré-codificado por uma matriz $\mathbf{G}$ de dimensão $N \times N$ que representa uma transformação linear arbitrária efetuada no transmissor. Este estágio pode assumir uma entre duas funções:

$$
\mathbf{G}=\left\{\begin{array}{cl}
\mathbf{I}_{N} ; & \text { se for } \mathrm{SC} \\
\mathbf{W}_{N}^{\mathcal{H}} ; & \text { se for OFDM }
\end{array}\right.
$$

Onde $\mathbf{I}_{N}$ é matriz identidade de tamanho $N$, e $\mathbf{W}_{N}^{\mathcal{H}}$ é a IDFT de $N$ pontos, normalizada tal que $\mathbf{W}_{N}^{\mathcal{H}} \mathbf{W}_{N}=\mathbf{I}_{N}$ e onde $(.)^{\mathcal{H}}$ denota conjugado transposto. A seguir, a matriz $\mathbf{V}^{T}$ insere uma faixa de guarda de $L$ símbolos ao final no bloco codificado $\mathbf{s}(n)$, montando o bloco $\mathbf{d}(n)$ de tamanho $M$, onde $M=N+L$. A faixa de guarda $L$ deve ser maior ou igual a memória do canal para evitar interferência entre blocos (IEB). Para o caso de zero-padding, considerado neste trabalho, a matriz $\mathbf{V}^{T}$ é da forma

$$
\mathbf{V}^{T}=\left[\begin{array}{c}
\mathbf{I}_{N} \\
\ldots \ldots \ldots \\
\mathbf{0}_{L \times N}
\end{array}\right]_{M \times N} .
$$

onde $\mathbf{0}_{u \times v}$ denota matriz nula de dimensão $u \times v$. Assim,

$$
\begin{aligned}
\mathbf{d}(n) & =\mathbf{V}^{T} \mathbf{s}(n) \\
& =[\underbrace{b_{1}(n), b_{2}(n), \ldots, b_{N}(n)}_{\mathbf{s}(n)}, \underbrace{0,0, \ldots, 0}_{L \text { zeros }}]^{T} .
\end{aligned}
$$

O bloco é então transmitido por um canal multipercurso, modelado neste trabalho como um filtro FIR de $P$ taps cujos coeficientes são amostras da envoltória complexa da resposta impulsional do canal. Supondo que durante o $n$-ésimo bloco a resposta impulsional permanece constante, i.e. $\mathbf{h}(n)=$ $\left[h_{0}(n), h_{1}(n), \ldots, h_{P-1}(n)\right]^{T}$, a transmissão pelo canal multipercurso pode ser representada por uma matriz de convolução Toeplitz triangular inferior $\mathbf{H}_{T}(n)$, que tem na primeira coluna o vetor do canal $\mathbf{h}(n)$, preenchido por zeros nas $M-P$ componentes restantes. As últimas $L$ colunas de $\mathbf{H}_{T}(n)$ não têm contribuição alguma no resultado da convolução, pois o vetor $\mathbf{d}(n)$ possui zeros nas suas últimas $L$ componentes. Isso posto, podemos alterar essas colunas de $\mathbf{H}_{T}(n)$ de maneira que ela se torne uma matriz circulante $\mathbf{H}_{C}(n)$, o que significa dizer que a convolução linear equivale a uma convolução circular.

\section{B. Recepção}

O sinal recebido é corrompido pelo vetor complexo de ruído aditivo Gaussiano branco $\mathbf{n}_{w}(n)$ na entrada do equipamento receptor. A matriz de covariância do ruído é

$$
\mathbf{K}_{\mathbf{n}_{w}}=\mathbb{E}\left[\mathbf{n}_{w}(n) \mathbf{n}_{w}^{\mathcal{H}}(n)\right]=\sigma_{n_{w}}^{2} \mathbf{I}_{M}
$$

onde $\sigma_{n_{w}}^{2}$, é a variância das componentes do vetor de ruído.O sinal recebido é então representado pelo vetor de dimensão $M \mathbf{x}(n)=\mathbf{H}_{C}(n) \mathbf{d}(n)+\mathbf{n}_{w}(n)$. Uma DFT de $M$-pontos é realizada sobre o vetor recebido $\mathbf{x}(n)$. Assim,

$$
\tilde{\mathbf{r}}(n)=\mathbf{W}_{M} \mathbf{H}_{C}(n) \mathbf{d}(n)+\mathbf{W}_{M} \mathbf{n}_{w}(n) .
$$

Como $\mathbf{W}_{M}$ é uma matriz unitária, podemos fazer

$$
\tilde{\mathbf{r}}(n)=\underbrace{\mathbf{W}_{M} \mathbf{H}_{C}(n) \mathbf{W}_{M}^{\mathcal{H}}}_{\tilde{\mathbf{Q}}_{D}(n)} \underbrace{\mathbf{W}_{M} \mathbf{d}(n)}_{\tilde{\mathbf{d}}(n)}+\mathbf{W}_{M} \mathbf{n}_{w}(n) .
$$

Utilizando propriedades matriciais, pode ser verificado que $\tilde{\mathbf{Q}}_{D}(n)$ é uma matriz diagonal, de dimensão $M \times M$, contendo na diagonal principal, amostras da resposta em freqüência do canal $\mathbf{q}(n)=\sqrt{M} \mathbf{W}_{M, P} \mathbf{h}(n)$, onde $\mathbf{W}_{M, P}$ é uma matriz $M \times P$ que contém as $P$ primeiras colunas da matriz $\mathbf{W}_{M}$. $\mathrm{O}$ vetor recebido $\tilde{\mathbf{r}}(n)$ é então equalizado por $M$ filtros 
independentes de 1 tap (ou filtro forward) organizados no vetor $\tilde{\mathbf{a}}(n)$, gerando na saída

$$
\tilde{\mathbf{z}}(n)=\tilde{\mathbf{A}}(n) \tilde{\mathbf{r}}(n)=\tilde{\mathbf{R}}(n) \tilde{\mathbf{a}}(n)
$$

onde $\tilde{\mathbf{R}}(n)=\operatorname{diag}(\tilde{\mathbf{r}}(n))$ e $\tilde{\mathbf{A}}(n)=\operatorname{diag}(\tilde{\mathbf{a}}(n))$. As deduções para os coeficientes deste filtro se encontram nas Seções III e IV. Efetua-se uma transformada inversa de $M$ pontos

$$
\mathbf{z}(n)=\mathbf{W}_{M}^{\mathcal{H}} \tilde{\mathbf{z}}(n) .
$$

Em seqüência, retiram-se os símbolos redundantes inseridos como faixa de guarda (neste caso, os $L$ zeros do fim do bloco), fazendo

$$
\mathbf{y}(n)=\mathbf{V} \mathbf{z}(n) .
$$

No caso de sistemas OFDM e/ou sistemas SC-FDE, o vetor $\mathbf{y}(\mathrm{n})$ é processado por $\mathbf{G}^{\mathcal{H}}$, dado por

$$
\mathbf{G}^{\mathcal{H}}=\left\{\begin{array}{cl}
\mathbf{I}_{N} ; & \text { se for SC } \\
\mathbf{W}_{N} ; & \text { se for OFDM }
\end{array}\right.
$$

e a saída é entregue para um decisor paralelo, que efetua detecção de mínima distância sobre todo o bloco em questão. Caso queiramos utilizar o estágio de DFE para os os sistemas SC-FDE, então uma conversão paralelo-serial precisa ser feita em cima do bloco $\mathbf{y}(\mathrm{n})$. Mais sobre o estágio de DFE na seção IV.

\section{EQUALIZAÇÃo LinEAR AdAPTATIVA RLS}

Nesta Seção, derivamos a expressões para o filtro linear $(L E)$ que opera no domínio da frequiência e serve tanto para o OFDM quanto para o SC-FDE. Define-se o vetor de erro como:

$$
\tilde{\mathbf{e}}_{L E}(n)=\tilde{\mathbf{d}}(i)-\tilde{\mathbf{R}}(i) \tilde{\mathbf{a}}(n)
$$

Neste algoritmo adaptativo, minimizamos a soma dos erros médio quadráticos ponderados exponencialmente. Define-se então a função custo a ser minimizada como:

$$
J_{L E}(n)=\sum_{i=1}^{n} \lambda^{n-i} \tilde{\mathbf{e}}_{L E}^{\mathcal{H}}(i) \tilde{\mathbf{e}}_{L E}(i) .
$$

onde $\lambda$ é um fator de esquecimento. No caso em que $\lambda=1$, não fazemos nenhuma distinção do passado, dando à ele o mesmo peso do presente na medida a ser minimizada. Por outro lado, podemos fazer com que $\lambda$ assuma valores muito próximos da unidade (porém menores). Isto levará o algoritmo a "esquecer" passado, isto é, dar menor peso as observações mais antigas. Esta maneira de ponderar as observações é indicada no rastreamento de canais variantes no tempo e será ilustrada mais a frente. Seguindo com o desenvolvimento do algoritmo e substituindo (10) em (11), obtemos:

$$
\begin{aligned}
J_{L E}(n) & =\sum_{i=1}^{n} \lambda^{n-i}\left[\tilde{\mathbf{d}}^{\mathcal{H}}(i) \tilde{\mathbf{d}}(i)-\tilde{\mathbf{d}}^{\mathcal{H}}(i) \tilde{\mathbf{R}}(i) \tilde{\mathbf{a}}(n)\right. \\
& \left.-\tilde{\mathbf{a}}^{\mathcal{H}}(n) \tilde{\mathbf{R}}^{\mathcal{H}}(i) \tilde{\mathbf{d}}(i)+\tilde{\mathbf{a}}^{\mathcal{H}}(n) \tilde{\mathbf{R}}^{\mathcal{H}}(i) \tilde{\mathbf{R}}(i) \tilde{\mathbf{a}}(n)\right](12)
\end{aligned}
$$

Calculando o gradiente da equação (12) com relação a $\mathbf{a}(n)$, chegamos à:

$$
\boldsymbol{\nabla}\left[J_{L E}(n)\right]=\sum_{i=1}^{n} \lambda^{n-i}\left[\tilde{\mathbf{R}}(i) \tilde{\mathbf{R}}^{*}(i) \tilde{\mathbf{a}}^{*}(n)-\tilde{\mathbf{R}}(i) \tilde{\mathbf{d}}^{*}(i)\right] .
$$

Queremos achar um ponto de mínimo para (12). Por isso vamos igualá-la à zero. Assim, e aproveitando para conjugar as expressões, de maneira que a resposta seja em função de $\tilde{\mathbf{a}}(n)$ :

$$
\sum_{i=1}^{n} \lambda^{n-i}\left[\tilde{\mathbf{R}}^{*}(i) \tilde{\mathbf{R}}(i)\right] \tilde{\mathbf{a}}(n)=\sum_{i=1}^{n} \lambda^{n-i}\left[\tilde{\mathbf{R}}^{*}(i) \tilde{\mathbf{d}}(i)\right]
$$

Identifica-se uma matriz de autocorrelação determinística no primeiro membro da equação (14):

$$
\boldsymbol{\Phi}(n)=\sum_{i=1}^{n} \lambda^{n-i}\left[\tilde{\mathbf{R}}^{*}(i) \tilde{\mathbf{R}}(i)\right] .
$$

Já no segundo membro da equação (14), temos um vetor $M \times 1$ de correlação cruzada determinística entre o vetor observado na recepção e o vetor transmitido

$$
\boldsymbol{\beta}(n)=\sum_{i=1}^{n} \lambda^{n-i}\left[\tilde{\mathbf{R}}^{*}(i) \tilde{\mathbf{d}}(i)\right]
$$

Podemos então escrever a solução de ã $(n)$ como

$$
\tilde{\mathbf{a}}(n)=[\boldsymbol{\Phi}(n)]^{-1} \boldsymbol{\beta}(n),
$$

onde $\boldsymbol{\Phi}(n)$ é uma matriz diagonal, visto que é obtida a partir do produto de duas matrizes diagonais. Podemos calcular os termos de maneira recursiva, observando a mesma estratégia tanto na equação (15) como em (16). Para isso, isola-se o termo em que $i=n$ :

$$
\begin{aligned}
\boldsymbol{\Phi}(n) & =\lambda\left\{\sum_{i=1}^{n-1} \lambda^{n-1-i}\left[\tilde{\mathbf{R}}^{*}(i) \tilde{\mathbf{R}}(i)\right]\right\}+\tilde{\mathbf{R}}^{*}(n) \tilde{\mathbf{R}}(n) \\
\boldsymbol{\Phi}(n) & =\lambda \boldsymbol{\Phi}(n-1)+\tilde{\mathbf{R}}^{*}(n) \tilde{\mathbf{R}}(n)
\end{aligned}
$$

De forma análoga, obtém-se

$$
\boldsymbol{\beta}(n)=\lambda \boldsymbol{\beta}(n-1)+\tilde{\mathbf{R}}^{*}(n) \tilde{\mathbf{d}}(n) .
$$

\section{EQUALIZAÇÃo COM DECISÃo REALIMENTADA ADAPTATIVA RLS}

Esta estrutura é utilizada apenas nos sistemas SC-FDE, uma vez que quando a equalização forward é imperfeita, i.e. $\tilde{\mathbf{A}}(n) \tilde{\mathbf{Q}}_{D} \neq \mathbf{I}_{M}$ há interferência intersimbólica no seqüência $\mathbf{y}(n)$ (ver Fig. 1), considerando-se o canal constante durante a duração de um bloco. Definimos o erro como uma função conjunta dos filtros forward (linear) e de feedback (retorno):

$$
\mathbf{e}_{D F E}(n)=\mathbf{V} \mathbf{W}^{\mathcal{H}} \tilde{\mathbf{R}}(n) \tilde{\mathbf{a}}(n)-\mathbf{C}^{T} \mathbf{f}(n)-\mathbf{b}(n),
$$

onde os $N_{f}$ coeficientes do filtro de retorno são identificados por $\mathbf{f}(n)$ e $\mathbf{C}(n)$ é a matriz que contém todas as decisões feitas em cima de um determinado bloco $\mathbf{y}(n)$ (no caso do sistema estar operando no modo Decision-Directed) ou, dos próprios símbolos transmitidos, no caso de seqüências de treinamento. Esta matriz é assim definida:

$$
\mathbf{C}(n)=\left[\mathbf{m}_{0}(n), \mathbf{m}_{1}(n), \ldots, \mathbf{m}_{N}(n)\right],
$$

onde os vetores $\mathbf{m}_{l}(n)$ são dados por

$$
\mathbf{m}_{l}(n)=\left[b_{l-1}(n), b_{l-2}(n), \ldots, b_{l-N_{f}}(n)\right]^{T} ; \quad l=1,2 \ldots, N .
$$


Chegamos agora ao ponto de desenvolver o método de mínimos quadrados para o sistema com o filtro de feedback. A nova função custo a ser minimizada (novamente, via RLS):

$$
J_{D F E}(n)=\sum_{i=1}^{n} \lambda^{n-i}\left\|\mathbf{e}_{D F E}(i)\right\|^{2} .
$$

A expressão acima também pode ser vista como uma convolução entre uma seqüência com decaimento exponencial $\left(\lambda^{n-i}\right)$ e o valor quadrático instantâneo do erro, definido em (20). Desenvolvendo a expressão (23), encontramos:

$$
\begin{aligned}
J & =\sum_{i=1}^{n} \lambda^{n-i}\left\{\tilde{\mathbf{a}}^{T} \tilde{\mathbf{R}}^{T}(i) \mathbf{W}^{\mathcal{H}} \mathbf{V}^{T}\left[\mathbf{V} \mathbf{W}^{T} \tilde{\mathbf{R}}^{*}(i) \tilde{\mathbf{a}}^{*}-\mathbf{C}^{\mathcal{H}}(i) \mathbf{f}^{*}-\mathbf{b}^{*}(i)\right]\right. \\
& -\mathbf{f}^{T} \mathbf{C}(i)\left[\mathbf{V} \mathbf{W}^{T} \tilde{\mathbf{R}}^{*}(i) \tilde{\mathbf{a}}^{*}-\mathbf{C}^{\mathcal{H}}(i) \mathbf{f}^{*}-\mathbf{b}^{*}(i)\right] \\
& \left.-\left[\tilde{\mathbf{a}}^{\mathcal{H}} \tilde{\mathbf{R}}^{\mathcal{H}}(i) \mathbf{W} \mathbf{V}^{T}-\mathbf{f}^{\mathcal{H}} \mathbf{C}^{*}(i)-\mathbf{s}^{\mathcal{H}}(i)\right] \mathbf{b}(i)\right\}
\end{aligned}
$$

Calculando o gradiente em relação aos dois filtros (forward e de feedback):

$\boldsymbol{\nabla}_{\mathbf{a}} J=\sum_{i=1}^{n} \lambda^{n-i} \boldsymbol{\Lambda}(i)\left\{\boldsymbol{\Lambda}^{\mathcal{H}}(i) \tilde{\mathbf{a}}^{*}(n)-\mathbf{C}^{\mathcal{H}}(i) \mathbf{f}^{*}(n)-\mathbf{b}^{*}(i)\right\}$

$\boldsymbol{\nabla}_{\mathbf{f}} J=\sum_{i=1}^{n} \lambda^{n-i} \mathbf{C}(i)\left\{\mathbf{C}^{\mathcal{H}}(i) \mathbf{f}^{*}(n)-\boldsymbol{\Lambda}^{\mathcal{H}}(i) \tilde{\mathbf{a}}^{*}(n)+\mathbf{b}^{*}(i)\right\}$

onde

$$
\boldsymbol{\Lambda}(n)=\tilde{\mathbf{R}}^{T}(n) \mathbf{W} \mathbf{V}^{T}
$$

Igualando os gradientes a zero e conjugando os termos, obtémse:

$\sum_{i=1}^{n} \lambda^{n-i} \boldsymbol{\Lambda}^{*}(i)\left\{\boldsymbol{\Lambda}^{\boldsymbol{T}}(i) \tilde{\mathbf{a}}(n)-\mathbf{C}^{T}(i) \mathbf{f}(n)\right\}=\sum_{i=1}^{n} \lambda^{n-i} \boldsymbol{\Lambda}^{*}(i) \mathbf{b}(i)$

$\sum_{i=1}^{n} \lambda^{n-i} \mathbf{C}^{*}(i)\left\{\boldsymbol{\Lambda}^{T}(i) \tilde{\mathbf{a}}(n)-\mathbf{C}^{T}(i) \mathbf{f}(n)\right\}=\sum_{i=1}^{n} \lambda^{n-i} \mathbf{C}^{*}(i) \mathbf{b}(i)$

Definindo

$$
\mathbf{T}^{*}(n)=\left[\begin{array}{c}
\boldsymbol{\Lambda}^{*}(n) \\
\cdots \\
\mathbf{C}^{*}(n)
\end{array}\right]
$$

podemos escrever (28) e (29) de uma forma mais compacta

$$
\underbrace{\sum_{i=1}^{n} \lambda^{n-i} \mathbf{T}^{*}(i) \mathbf{T}^{\mathcal{H}}(i)}_{\boldsymbol{\Phi}(n)} \mathbf{v}(n)=\underbrace{\sum_{i=1}^{n} \lambda^{n-i} \mathbf{T}^{*}(i) \mathbf{b}(i)}_{\boldsymbol{\beta}(\boldsymbol{n})},
$$

em que os coeficientes dos filtros forward e feedback foram agrupados num só vetor

$$
\mathbf{v}(n)=\left[\begin{array}{c}
\tilde{\mathbf{a}}(n) \\
\cdots \\
\mathbf{f}(n)
\end{array}\right]
$$

e onde sua solução é encontrada fazendo

$$
\mathbf{v}(n)=[\boldsymbol{\Phi}(n)]^{-1} \boldsymbol{\beta}(n),
$$

sendo $\boldsymbol{\Phi}(\boldsymbol{n})$ uma matriz de dimensão $\left(M+N_{f}\right) \times\left(M+N_{f}\right)$. A sua atualização é dada por:

$$
\boldsymbol{\Phi}(n)=\lambda \mathbf{\Phi}(n-1)+\mathbf{T}^{*}(n) \mathbf{T}^{\mathcal{H}}(n),
$$

ao passo que

$$
\boldsymbol{\beta}(n)=\lambda \boldsymbol{\beta}(n-1)+\mathbf{T}^{*}(n) \mathbf{b}(n) .
$$

\section{Resultados}

Os resultados de simulação apresentados são para sistemas de transmissão por blocos SC e OFDM que utilizam modulação QPSK. Os blocos foram escolhidos de tamanho $N=16$ neste projeto. O canal de comunicações é modelado por um filtro FIR com $P=4$ taps, cujos coeficientes são do tipo

$$
h_{k}=p_{k} \alpha_{k} ; \quad k=1,2, \ldots, P
$$

onde $\left\{\alpha_{k}\right\}_{k=1}^{P}$ são variáveis aleatórias Gaussianas complexas, estatisticamente independentes, de média nula e com $\mathbb{E}\left[\left|\alpha_{k}\right|^{2}\right]=1$, no caso de canal fixo (no tempo). Os valores de $\alpha_{k}$ são sorteados no início de cada simulação e mantidos fixos ao longo de cada teste. Os pesos $p_{k}$ satisfazem a $\sum_{k=1}^{P} p_{k}^{2}=1$. Para canal variante no tempo, supõe-se que os $P$ coeficientes do canal são dados por

$$
h_{k}(n)=p_{k} \gamma_{k}(n) ; \quad k=1,2, \ldots, P
$$

onde $\left\{\gamma_{k}(n)\right\}_{k=1}^{P}, n=1,2, \ldots$, são seqüências independentes de variáveis aleatórias Gaussianas correlatadas obtidas ao filtrar-se um processo Gaussiano complexo branco por um filtro cuja resposta é dada aproximadamente por [6]

$$
H_{d o p}(f)=\frac{K}{\sqrt{1-\left(\frac{f}{f_{d}}\right)^{2}}},
$$

onde $K$ é uma constante de normalização, $f_{d}=\frac{v}{\lambda_{w}}$ é o máximo desvio doppler de freqüência, $\lambda_{w}$ é o comprimento de onda e $v$ é a velocidade de deslocamento do terminal móvel. Os resultados para canal variante no tempo são mostrados em termos da freqüência doppler normalizada $\left(f_{d} T\right)$, onde $T$ é o intervalo de 1 bloco. Os pesos utilizados nas simulações foram $\mathbf{p}=[0.8677,0.4339,0.2169,0.1085]^{T}$. A faixa de guarda para cada bloco é $L=4$. Na Figura 2 estão apresentadas as curvas de desempenho de taxa de erro de bit para os sistemas SC empregando FDE-LE e FDE-DFE e para o sistema OFDM. Foram utilizados 50 blocos de treinamento, após os quais os sistemas foram colocados em modo de operação decision direct, com 10.000 realizações. As curvas mostram um ganho evidente dos sistemas SC-FDE em relação ao OFDM, especialmente quando considera-se o estágio DFE. Comparando-se os sistemas SC, nota-se que a utilização de um estágio DFE apresenta ganho da ordem de 2 a $3 \mathrm{~dB}$. Na Figura 3 estão apresentadas as curvas de desempenho da taxa de erro de bit para os sistemas SC-FDE-DFE utilizando um equalizador de retorno com 3 taps e um sistema OFDM. O canal apresenta $f_{d} T=0.001$. Novamente, o sistema SC-FDE-DFE apresentou desempenho superior com um piso de BER bem inferior ao apresentado pelo OFDM. 


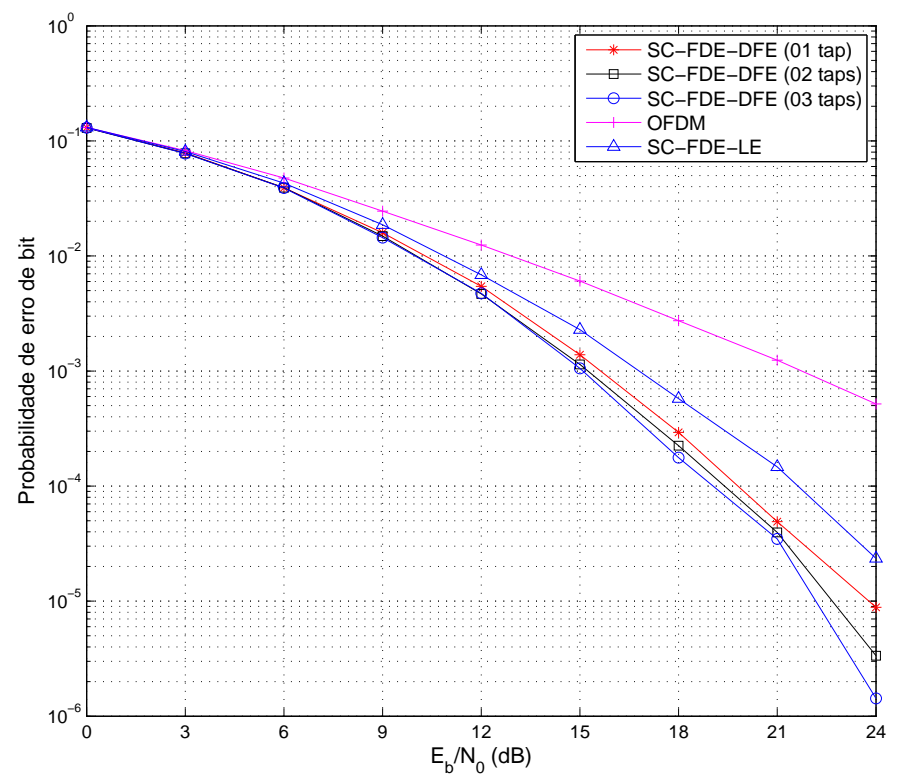

Fig. 2. BER comparativa entre os sistemas considerados (canal fixo)

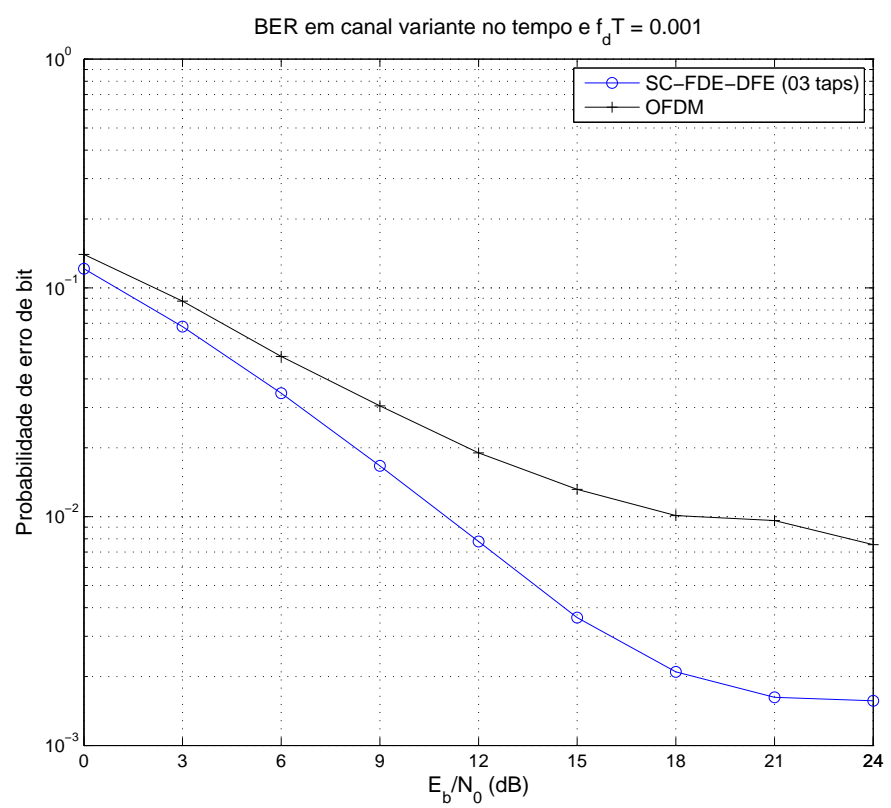

Fig. 3. OFDM versus SC-FDE-DFE em canal variante no tempo, $f_{d} T=$ 0.001

\section{CONCLUSÕES}

Pelos resultados das simulações, fica evidente o ganho dos sistemas SC-FDE em relação ao OFDM, especialmente quando considera-se o estágio de retorno ou DFE. Sua implementação, contudo, é computacionalmente mais pesada, uma vez que há processamento símbolo-à-símbolo sobre o bloco que é entregue ao decisor. OFDM e SC-FDE-LE tem processamento em bloco, sendo mais rápidos e mais fáceis de projetar, porém com desempenho inferior.

\section{REFERÊNCIAS}

[1] D. Falconer, S. L. Ariyavisitakul, A. Benyamin-Seeyar, and B. Eidson, "Frequency domain equalization for single-carrier broadband wireless systems," IEEE Communications Magazine, 2002.

[2] J. Proakis, Digital Communications. McGraw-Hill, 1995.

[3] M. V. Clark, "Adaptive frequency-domain equalization and diversity combining for broadband wireless communications," IEEE JSAC, vol. 16, pp. 1385-1395, Outubro 1998.

[4] Z. Wang and G. B. Giannakis, "Wireless multicarrier communications: Where Fourier meets Shannon,” IEEE Signal Processing Mag., pp. 29-47, May 2000.

[5] N. Benvenuto and S. Tomasin, "On the comparison between OFDM and single carrier modulation with a DFE using a frequency-domain feedforward filter," IEEE Trans. Commun., vol. 50, pp. 947-955, Junho 2002.

[6] T. S. Rappaport, Wireless Communications: Principles and Practice. Prentice Hall PTR, 1996. 